\title{
On the roots of generalized Wills $\mu$-polynomials
}

\author{
María A. Hernández Cifre and Jesús Yepes Nicolás
}

\begin{abstract}
We investigate the roots of a family of geometric polynomials of convex bodies associated to a given measure $\mu$ on the non-negative real line $\mathbb{R}_{\geq 0}$, which arise from the so called Wills functional. We study its structure, showing that the set of roots in the upper half-plane is a closed convex cone, containing the non-positive real axis $\mathbb{R}_{\leq 0}$, and strictly increasing in the dimension, for any measure $\mu$. Moreover, it is proved that the 'smallest' cone of roots of these $\mu$-polynomials is the one given by the Steiner polynomial, which provides, for example, additional information about the roots of $\mu$-polynomials when the dimension is large enough. It will also give necessary geometric conditions for a sequence $\left\{m_{i}: i=0,1, \ldots\right\}$ to be the moments of a certain measure on $\mathbb{R}_{\geq 0}$, a question regarding the so called (Stieltjes) moment problem.
\end{abstract}

\section{Introduction and main results}

Let $\mathcal{K}^{n}$ be the set of all convex bodies, i.e., compact convex sets, in the $n$ dimensional Euclidean space $\mathbb{R}^{n}$, and let $B_{n}$ be the $n$-dimensional unit ball. The volume of a set $M \subsetneq \mathbb{R}^{n}$, i.e., its $n$-dimensional Lebesgue measure, is denoted by $\operatorname{vol}(M)$ and, in particular, we write $\kappa_{n}=\operatorname{vol}\left(B_{n}\right)$. Finally, with $\operatorname{bd} M, \operatorname{lin} M$, aff $M$, int $M$ and relint $M$ we represent its boundary, linear hull, affine hull, interior and relative interior, respectively. For convex bodies $K, E \in \mathcal{K}^{n}$ and a non-negative real number $\lambda$, the well-known Steiner formula states that the volume of the Minkowski sum $K+\lambda E$ can be expressed as a polynomial of degree (at most) $n$ in the parameter $\lambda$,

$$
\operatorname{vol}(K+\lambda E)=\sum_{i=0}^{n}\left(\begin{array}{l}
n \\
i
\end{array}\right) \mathrm{W}_{i}(K ; E) \lambda^{i} ;
$$

here the coefficients $\mathrm{W}_{i}(K ; E)$ are the relative quermassintegrals of $K$ with respect to $E$, and they are a special case of the more general defined mixed volumes (see e.g. Section 5.1 in [17]). In particular, $\mathrm{W}_{0}(K ; E)=\operatorname{vol}(K)$ and $\mathrm{W}_{n}(K ; E)=\operatorname{vol}(E)$.

Mathematics Subject Classification (2010): Primary 52A20, 52A39; Secondary 30C15, 28A25.

Keywords: Generalized Wills functional, location of roots, weighted Steiner polynomial, measures, Stieltjes moment problem. 
In 1973 ([20]), Wills introduced and studied the functional

$$
W(K)=\sum_{i=0}^{n}\left(\begin{array}{l}
n \\
i
\end{array}\right) \frac{W_{i}\left(K ; B_{n}\right)}{\kappa_{i}}
$$

because of its possible relation with the so called lattice-point enumerator

$$
G(K)=\#\left(K \cap \mathbb{Z}^{n}\right),
$$

and conjectured that $W(K)$ bounded by above $G(K)$. Although Hadwiger [6] showed that Wills' conjecture was wrong (see also [1]), the Wills functional turned out to have several interesting applications, e.g., in discrete geometry, where there exist nice relations of this functional with the so called successive minima of a convex body, or in deriving exponential moment inequalities for Gaussian random processes [19]. Many other nice properties of this functional, as well as relations with other measures, have been studied in the last years, see e.g. [5], [6], [15], [20], [21], and $[22]$.

In particular, in [5] Hadwiger showed, among others, the following integral representations of $W(K)$ :

$$
W(K)=\int_{\mathbb{R}^{n}} e^{-\pi d(x, K)^{2}} \mathrm{~d} x, \quad W(K)=2 \pi \int_{0}^{\infty} \operatorname{vol}\left(K+t B_{n}\right) t e^{-\pi t^{2}} \mathrm{~d} t,
$$

where $d(x, K)$ denotes the Euclidean distance between $x \in \mathbb{R}^{n}$ and $K$.

Recently, Kampf [10] has proved that generalizations of the above relations remain true when the 'distance' $d_{E}(x, K)$, between $x \in \mathbb{R}^{n}$ and $K$, relative to a convex body $E$ with $0 \in \operatorname{int} E$ is considered, i.e.,

$$
\int_{\mathbb{R}^{n}} e^{-\pi d_{E}(x, K)^{2}} \mathrm{~d} x=2 \pi \int_{0}^{\infty} \operatorname{vol}(K+t E) t e^{-\pi t^{2}} \mathrm{~d} t=\sum_{i=0}^{n}\left(\begin{array}{c}
n \\
i
\end{array}\right) \frac{\mathrm{W}_{i}(K ; E)}{\kappa_{i}} .
$$

Moreover, a more general functional can be obtained replacing $e^{-\pi t^{2}}$ by a function $G(t)$ which is properly associated to a measure $\mu$ on the non-negative real line $\mathbb{R}_{\geq 0}$ :

$$
\int_{\mathbb{R}^{n}} G\left(d_{E}(x, K)\right) \mathrm{d} x \quad \text { with } G(t)=\mu([t, \infty)) .
$$

We extend this functional to any pair of convex bodies $K$ and $E$, allowing gauge bodies $E$ with dimension $\operatorname{dim} E<n$.

To this aim, for a given $E \in \mathcal{K}^{n}$ with $0 \in \operatorname{relint} E$ and $x-y \in \operatorname{lin} E$, let $d_{E}(x, y)=\inf \{\lambda \geq 0: x-y \in \lambda E\}$. We notice that if $E$ is 0-symmetric, i.e., $E=-E$, this function defines a distance on $\operatorname{lin} E$. Then, for $x \in K+\operatorname{lin} E$, we have

$$
d_{E}(x, K)=\inf \left\{d_{E}(x, y): y \in K \cap(x+\operatorname{lin} E)\right\}=\inf \{r \geq 0: x \in K+r E\} .
$$

Thus, the expression $d_{E}(x, K)$ is only defined for $x \in K+\operatorname{lin} E$ and, following the idea used in [10], the next result is fulfilled. It will be shown in Section 2. 
Lemma 1.1. Let $\mu$ be a (finite) measure on $\mathbb{R}_{\geq 0}$ such that the moments $m_{i}(\mu)=$ $\int_{0}^{\infty} t^{i} \mathrm{~d} \mu(t)$ of $\mu, i=0, \ldots, n$, exist and are finite, and let $G(t)=\mu([t, \infty))$, $t \in \mathbb{R}_{\geq 0}$. Then, for $K, E \in \mathcal{K}^{n}$ with $0 \in \operatorname{relint} E$, we have that

$$
\int_{K+\operatorname{lin} E} G\left(d_{E}(x, K)\right) \mathrm{d} x=\int_{0}^{\infty} \operatorname{vol}(K+t E) \mathrm{d} \mu(t)=\sum_{i=0}^{n}\left(\begin{array}{c}
n \\
i
\end{array}\right) \mathrm{W}_{i}(K ; E) m_{i}(\mu) .
$$

We observe that the right-hand side in the last equality is translation invariant. Thus, for any convex bodies $K, E \in \mathcal{K}^{n}$, any $x_{0} \in \operatorname{relint} E$, and a given measure $\mu$ on $\mathbb{R}_{\geq 0}$, we have a Wills type functional (associated to $\mu$ )

$$
\mathcal{W}^{\mu}(K ; E)=\int_{K+\operatorname{lin}\left(E-x_{0}\right)} G\left(d_{E-x_{0}}(x, K)\right) \mathrm{d} x=\sum_{i=0}^{n}\left(\begin{array}{l}
n \\
i
\end{array}\right) \mathrm{W}_{i}(K ; E) m_{i}(\mu) .
$$

Thus, we can always assume, without loss of generality, that $0 \in \operatorname{relint} E$.

Using the previous lemma for the function $G(t)=e^{-\pi t^{2}}$, we get the relative Wills functional for convex bodies $K, E \in \mathcal{K}^{n}$, namely,

$$
\mathcal{W}(K ; E)=\int_{K+\operatorname{lin} E} e^{-\pi d_{E}(x, K)^{2}} \mathrm{~d} x=\sum_{i=0}^{n}\left(\begin{array}{l}
n \\
i
\end{array}\right) \frac{\mathrm{W}_{i}(K ; E)}{\kappa_{i}} .
$$

In the case of the Steiner functional $\sum_{i=0}^{n}\left(\begin{array}{c}n \\ i\end{array}\right) \mathrm{W}_{i}(K ; E)$, we have the following $\mu$-type representation, which will be also shown in Section 2.

Theorem 1.1. Let $K, E \in \mathcal{K}^{n}$ with $0 \in \operatorname{relint} E$. Then

$$
\sum_{i=0}^{n}\left(\begin{array}{l}
n \\
i
\end{array}\right) \mathrm{W}_{i}(K ; E)=\lim _{\sigma \rightarrow 0^{+}} \int_{K+\operatorname{lin} E} \int_{d_{E}(x, K)}^{\infty} \frac{1}{\sqrt{2 \pi} \sigma} e^{-\frac{(t-1)^{2}}{2 \sigma^{2}}} \mathrm{~d} t \mathrm{~d} x .
$$

Moreover, such an expression (in which a non-discrete measure $\mu$ on $\mathbb{R}_{\geq 0}$ is considered) is only possible through a 'pass to the limit' process.

In the following we will write, for $K, E \in \mathcal{K}^{n}$ and a measure $\mu$ on $\mathbb{R}_{\geq 0}$,

$$
f_{K ; E}^{\mu}(z)=\sum_{i=0}^{n}\left(\begin{array}{l}
n \\
i
\end{array}\right) \mathrm{W}_{i}(K ; E) m_{i}(\mu) z^{i}
$$

to denote the Wills $\mu$-polynomial of $K$ with respect to $E$, regarded as a formal polynomial in a complex variable $z \in \mathbb{C}$. Similarly, we will represent the relative Steiner and Wills polynomials in a variable $z \in \mathbb{C}$ (cf. (1.1), (1.2)), respectively, by

$$
f_{K ; E}(z)=\sum_{i=0}^{n}\left(\begin{array}{c}
n \\
i
\end{array}\right) \mathrm{W}_{i}(K ; E) z^{i} \quad \text { and } \quad f_{K ; E}^{g}(z)=\sum_{i=0}^{n}\left(\begin{array}{c}
n \\
i
\end{array}\right) \frac{\mathrm{W}_{i}(K ; E)}{\kappa_{i}} z^{i} .
$$

From now on, we will denote by $g$ the measure associated to $G(t)=e^{-\pi t^{2}}$, which yields the (classical) Wills functional when $E=B_{n}$ (cf. (1.3)), and whose moments are $m_{i}(g)=1 / \kappa_{i}$. 
Motivated by previous works of Henk, Hernández Cifre and Saorín on the roots of the Steiner polynomial (see [7], [8]), here we are interested in studying properties of the roots of the above family of polynomials $f_{K ; E}^{\mu}(z)$. To this end, we fix the notation which will be used along the paper. Denoting by $\operatorname{Re} z, \operatorname{Im} z$ and $\arg z$, the real part, imaginary part and the principal argument of a complex number $z$, respectively, let $\mathbb{C}^{+}=\{z \in \mathbb{C}: \operatorname{Im} z \geq 0\}$ be the set of complex numbers with non-negative imaginary part, and for a fixed measure $\mu$, let

$$
\mathcal{R}^{\mu}(n)=\left\{z \in \mathbb{C}^{+}: f_{K ; E}^{\mu}(z)=0 \quad \text { for } \quad K, E \in \mathcal{K}^{n}, \operatorname{dim}(K+E)=n\right\}
$$

be the set of all roots of $f_{K ; E}^{\mu}(z), K, E \in \mathcal{K}^{n}$, in the upper half-plane.

Theorem 1.2. For any measure $\mu, \mathcal{R}^{\mu}(n)$ is a convex cone, containing the nonpositive real axis $\mathbb{R}_{\leq 0}$.

We notice that in order to construct these Wills type functionals, we work with a measure $\mu$ on $\mathbb{R}_{\geq 0}$ (cf. Lemma 1.1). The results by Kampf [10] are stated in the more general setting when a signed measure $\rho$ is considered. We will show that in this case the corresponding set $\mathcal{R}^{\rho}(n)$, although it is always a cone, it is not, in general, convex (see Proposition 3.1).

According to the above theorem, the 'geometry' of the set $\mathcal{R}^{\mu}(n)$ is given by the 'upper ray' of the boundary. Regarding the possible inclusion of this ray in the cone and the monotonicity in the dimension, we have the following results, which will be shown in Section 3.

Theorem 1.3. For any measure $\mu$, the cone $\mathcal{R}^{\mu}(n)$ is closed.

Theorem 1.4. For any measure $\mu, \mathcal{R}^{\mu}(n)$ is strictly increasing in the dimension, i.e., $\mathcal{R}^{\mu}(n) \subsetneq \mathcal{R}^{\mu}(n+1)$.

Let $\mathcal{R}(n)=\left\{z \in \mathbb{C}^{+}: f_{K ; E}(z)=0\right.$ for $\left.K, E \in \mathcal{K}^{n}, \operatorname{dim}(K+E)=n\right\}$ be the set of roots of all Steiner polynomials $f_{K ; E}(z), K, E \in \mathcal{K}^{n}$, in the upper halfplane. Then there exists a relation between (the cone of) the roots of the Steiner polynomial and the Wills $\mu$-polynomials.

Theorem 1.5. For any measure $\mu$, it holds $\mathcal{R}(n) \subset \mathcal{R}^{\mu}(n)$. Moreover, if $\mu$ verifies $\mu\left(\mathbb{R}_{\geq 0} \backslash\left\{0, m_{i}(\mu) / m_{i-1}(\mu)\right\}\right) \neq 0$ for $i=1,2, \ldots$, the inclusion is strict.

This result shows that the 'smallest' cone of roots of Wills $\mu$-polynomials is the one given by the Steiner polynomial, which provides additional information about the roots of $\mu$-polynomials when the dimension is large enough, namely, that for any measure $\mu, \mathcal{R}^{\mu}(n)$ covers the whole upper half-plane $\mathbb{C}^{+}$, except $\mathbb{R}_{>0}$, when $n$ tends to infinity.

Corollary 1.1. If $n \geq 10$ it holds $\left\{z \in \mathbb{C}^{+}: \operatorname{Re} z \leq 0\right\} \subsetneq \mathcal{R}^{\mu}(n)$. Moreover, given $\gamma \in \mathbb{C}^{+} \backslash \mathbb{R}_{>0}$, there exists $n_{\gamma}$ such that $\gamma \in \mathcal{R}^{\mu}(n)$ for all $n \geq n_{\gamma}$. 
Similarly, we can determine the 'largest' cone of roots containing $\mathcal{R}^{\mu}(n)$ for any $\log$-concave measure $\mu$ on $\mathbb{R}_{\geq 0}$ (see Section 4 for the definition). Before stating the result, we need additional notation: given a sequence $\mathbf{m}=\left(m_{i}\right)_{i \in \mathbb{N}}, m_{i}>0$, let

$$
f_{K ; E}^{\mathbf{m}}(z)=\sum_{i=0}^{n}\left(\begin{array}{l}
n \\
i
\end{array}\right) \mathrm{W}_{i}(K ; E) m_{i} z^{i},
$$

$K, E \in \mathcal{K}^{n}$ with $\operatorname{dim}(K+E)=n$, and, according to our terminology, we denote the corresponding cone of roots by $\mathcal{R}^{\mathbf{m}}(n)$, i.e.,

$$
\mathcal{R}^{\mathbf{m}}(n)=\left\{z \in \mathbb{C}^{+}: f_{K ; E}^{\mathbf{m}}(z)=0 \quad \text { for } K, E \in \mathcal{K}^{n}, \operatorname{dim}(K+E)=n\right\} .
$$

Theorem 1.6. Let $\boldsymbol{\omega}=\left(i^{i}\right)_{i \in \mathbb{N}}=\left(1,1,2^{2}, \ldots\right)$. Then $\mathcal{R}^{\mu}(n) \subset \mathcal{R}^{\boldsymbol{\omega}}(n)$ for any log-concave measure $\mu$ on $\mathbb{R}_{\geq 0}$.

We observe that $f_{K ; E}^{\omega}(z)$ is a Wills $\mu$-type polynomial, because the 'weights' $m_{i}=i^{i}$ are the moments of a measure (see page 104 of [2] for a question regarding the so called (Stieltjes) moment problem; see also e.g. [13]).

We observe that properties collected in Theorem 1.5 and Corollary 1.1 are not true for general m-polynomials (see Remark 4.2). Thus, Theorem 1.5 and Corollary 1.1 state necessary 'geometric' conditions for the (Stieltjes) moment problem.

The above results will be shown in Section 4. Finally, in Section 5 we deal with the stability of this kind of polynomials, listing additional properties concerning the (relative) Wills polynomial $f_{K ; E}^{g}(z)$.

\section{On the Wills type functionals}

We start this section proving Lemma 1.1. We follow the idea of the proof given by Kampf in [10], which is based on Fubini's theorem. Here $\chi_{M}$ will denote the characteristic function of the set $M \subset \mathbb{R}^{n}$.

Proof of Lemma 1.1. Clearly, for $x \in K+\operatorname{lin} E$ we have $d_{E}(x, K) \leq t$ if and only if $x \in K+t E$. Using this property and Steiner formula (1.1), we get

$$
\begin{aligned}
\int_{K+\operatorname{lin} E} G\left(d_{E}(x, K)\right) \mathrm{d} x & =\int_{K+\operatorname{lin} E} \mu\left(\left[d_{E}(x, K), \infty\right)\right) \mathrm{d} x \\
& =\int_{\mathbb{R}^{n}} \int_{0}^{\infty} \chi_{\left\{y \in K+\operatorname{lin} E: d_{E}(y, K) \leq t\right\}}(x) \mathrm{d} \mu(t) \mathrm{d} x \\
& =\int_{\mathbb{R}^{n}} \int_{0}^{\infty} \chi_{K+t E}(x) \mathrm{d} \mu(t) \mathrm{d} x=\int_{0}^{\infty} \int_{\mathbb{R}^{n}} \chi_{K+t E}(x) \mathrm{d} x \mathrm{~d} \mu(t) \\
& =\int_{0}^{\infty} \operatorname{vol}(K+t E) \mathrm{d} \mu(t)=\sum_{i=0}^{n}\left(\begin{array}{c}
n \\
i
\end{array}\right) \mathrm{W}_{i}(K ; E) \int_{0}^{\infty} t^{i} \mathrm{~d} \mu(t) \\
& =\sum_{i=0}^{n}\left(\begin{array}{c}
n \\
i
\end{array}\right) \mathrm{W}_{i}(K ; E) m_{i}(\mu) .
\end{aligned}
$$

This shows the lemma. 
Next we show that the Steiner functional can be also obtained as a generalized Wills type functional for a particular 'limit' measure.

Proof of Theorem 1.1. Let $\mu_{\sigma}$ be the measure on $\mathbb{R}_{\geq 0}$ given by

$$
\mu_{\sigma}(A)=\int_{A} f_{\sigma}(t) \mathrm{d} t, \quad \text { with } \quad f_{\sigma}(t)=\frac{1}{\sqrt{2 \pi} \sigma} e^{-\frac{(t-1)^{2}}{2 \sigma^{2}}}
$$

(see Figure 1) and let $G_{\sigma}$ be the function

$$
G_{\sigma}(s)=\int_{s}^{\infty} \mathrm{d} \mu_{\sigma}(t)=\int_{s}^{\infty} f_{\sigma}(t) \mathrm{d} t .
$$

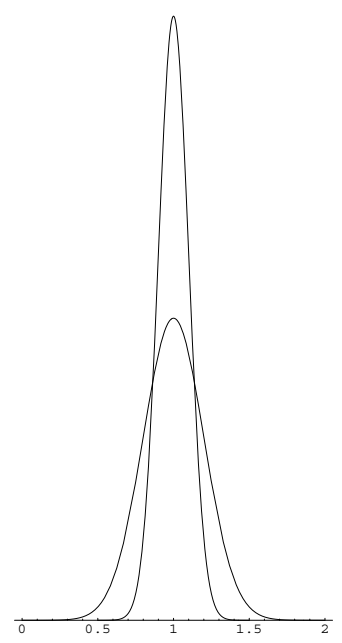

Figure 1. The function $f_{\sigma}(t)=\frac{1}{\sqrt{2 \pi} \sigma} e^{-\frac{(t-1)^{2}}{2 \sigma^{2}}}$ for $\sigma=0.2$ and $\sigma=0.1$.

On the one hand we observe that, denoting by $\bar{\mu}_{\sigma}$ the measure on $\mathbb{R}_{\leq 0}$ associated to the function $f_{\sigma}(t)$, we have

$$
\begin{aligned}
\lim _{\sigma \rightarrow 0^{+}} \int_{-\infty}^{0} t^{r} \mathrm{~d} \bar{\mu}_{\sigma}(t) & =\lim _{\sigma \rightarrow 0^{+}} \int_{-\infty}^{0} t^{r} f_{\sigma}(t) \mathrm{d} t \\
& =\lim _{\sigma \rightarrow 0^{+}} \int_{-\infty}^{-1 /(\sqrt{2} \sigma)} \frac{1}{\sqrt{\pi}}(\sqrt{2} \sigma s+1)^{r} e^{-s^{2}} \mathrm{~d} s \\
& =\lim _{\sigma \rightarrow 0^{+}} \int_{-\infty}^{-1 /(\sqrt{2} \sigma)} \frac{1}{\sqrt{\pi}}\left(\sum_{i=0}^{r}\left(\begin{array}{c}
r \\
i
\end{array}\right)(\sqrt{2} \sigma s)^{i}\right) e^{-s^{2}} \mathrm{~d} s \\
& =\frac{1}{\sqrt{\pi}} \sum_{i=0}^{r}\left(\begin{array}{c}
r \\
i
\end{array}\right) 2^{i / 2}\left(\lim _{\sigma \rightarrow 0^{+}} \sigma^{i} \int_{-\infty}^{-1 /(\sqrt{2} \sigma)} s^{i} e^{-s^{2}} \mathrm{~d} s\right)=0 .
\end{aligned}
$$


On the other hand, if $\varphi_{\sigma}(t)=e^{t+t^{2} \sigma^{2} / 2}$ denotes the moment generating function of a normal distribution $\mathrm{N}(1, \sigma)$ associated to the density function $f_{\sigma}$ (on the real line $\mathbb{R})$, then its $i$-th derivative can be written as

$$
\varphi_{\sigma}^{(i)}(t)=e^{t+t^{2} \sigma^{2} / 2}\left(1+t \sigma^{2}\right)^{i}+\sigma^{2} g_{\sigma}(t)
$$

for a suitable function $g_{\sigma}(t)$ bounded in a neighborhood of $\sigma=0$, and thus $\lim _{\sigma \rightarrow 0^{+}} \varphi_{\sigma}^{(i)}(0)=1$. So, by Lemma 1.1,

$$
\int_{K+\operatorname{lin} E} G_{\sigma}\left(d_{E}(x, K)\right) \mathrm{d} x=\sum_{i=0}^{n}\left(\begin{array}{c}
n \\
i
\end{array}\right) \mathrm{W}_{i}(K ; E) \int_{0}^{\infty} t^{i} \mathrm{~d} \mu_{\sigma}(t)
$$

and then

$$
\begin{aligned}
\lim _{\sigma \rightarrow 0^{+}} \int_{K+\operatorname{lin} E} G_{\sigma}\left(d_{E}(x, K)\right) \mathrm{d} x=\sum_{i=0}^{n}\left(\begin{array}{c}
n \\
i
\end{array}\right) \mathrm{W}_{i}(K ; E) \lim _{\sigma \rightarrow 0^{+}} \int_{0}^{\infty} t^{i} \mathrm{~d} \mu_{\sigma}(t) \\
=\sum_{i=0}^{n}\left(\begin{array}{c}
n \\
i
\end{array}\right) \mathrm{W}_{i}(K ; E)\left(\lim _{\sigma \rightarrow 0^{+}} \int_{0}^{\infty} t^{i} \mathrm{~d} \mu_{\sigma}(t)+\lim _{\sigma \rightarrow 0^{+}} \int_{-\infty}^{0} t^{i} \mathrm{~d} \bar{\mu}_{\sigma}(t)\right) \\
=\sum_{i=0}^{n}\left(\begin{array}{c}
n \\
i
\end{array}\right) \mathrm{W}_{i}(K ; E) \lim _{\sigma \rightarrow 0^{+}} \int_{-\infty}^{\infty} t^{i} f_{\sigma}(t) \mathrm{d} t \\
=\sum_{i=0}^{n}\left(\begin{array}{c}
n \\
i
\end{array}\right) \mathrm{W}_{i}(K ; E) \lim _{\sigma \rightarrow 0^{+}} \varphi_{\sigma}^{(i)}(0)=\sum_{i=0}^{n}\left(\begin{array}{c}
n \\
i
\end{array}\right) \mathrm{W}_{i}(K ; E),
\end{aligned}
$$

where, in the last equality but one, we have used the well known connection between the moments of a measure and its moment generating function (see e.g. Sections 2.3-2.4 and Theorem 2.3.7 in [3]).

Finally, the last assertion of the theorem follows from the fact that if for a measure $\widetilde{\mu}$ it holds $m_{i}(\widetilde{\mu})=1$ for all $i=0, \ldots, n$, with $n \geq 2$, then

$$
\int_{0}^{\infty}(t-1)^{2} \mathrm{~d} \widetilde{\mu}(t)=\int_{0}^{\infty} t^{2} \mathrm{~d} \widetilde{\mu}(t)-2 \int_{0}^{\infty} t \mathrm{~d} \widetilde{\mu}(t)+\int_{0}^{\infty} \mathrm{d} \widetilde{\mu}(t)=0,
$$

which implies that $\tilde{\mu}$ is a discrete measure concentrated at $t=1$.

\section{The cone of roots of Wills $\mu$-polynomials}

From now on and unless we explicitly say the opposite, we will always assume that, for a given measure $\mu$ on $\mathbb{R}_{\geq 0}$, its moments $m_{i}(\mu)>0$ for all $i \geq 0$, i.e., we omit the case when $\mu$ is discrete and concentrates the measure at $t=0$. We will also need the following additional notation. For convex bodies $K, E \in \mathcal{K}^{n}$ such that $f_{K ; E}^{\mu}(z)$ has a non-zero root let

$$
\theta_{K ; E}^{\mu}=\min \left\{\arg z: z \in \mathbb{C}^{+} \backslash\{0\}, f_{K ; E}^{\mu}(z)=0\right\},
$$


and we denote by

$$
\mathcal{R}^{\mu}(K ; E)=\left\{z \in \mathbb{C}^{+} \backslash\{0\}: \arg z \geq \theta_{K ; E}^{\mu}\right\} \cup\{0\}
$$

the convex cone, in the upper half-plane, generated as the positive hull of the roots of the polynomial $f_{K ; E}^{\mu}(z)$ and $\mathbb{R}_{\leq 0}$.

We start this section stating some preliminary lemmas which will be needed for the proof of Theorem 1.2. In Theorem 5.2 of [12] the following result is proved.

Theorem 3.1 (Kato, 1995). Let $\xi(t)$ be an unordered $n$-tuple of complex numbers, depending continuously on a real variable $t$ in a (closed or open) interval $I$. Then there exist $n$ continuous functions $\nu_{i}(t), i=1, \ldots, n$, which constitute the values of the $n$-tuple $\xi(t)$ for each $t \in I$.

As a consequence, we get the following lemma.

Lemma 3.1. Let $K(t) \in \mathcal{K}^{n}, t \in[a, b]$, be a one-parameter continuous (on $t$ ) family of convex bodies with $\operatorname{dim} K(a)=n-k-1$ and $\operatorname{dim} K(t)=n-k$ for all $t \in(a, b]$, and let $E \in \mathcal{K}^{n}$ with $\operatorname{dim} E=r>k$ and $\operatorname{dim}(K(t)+E)=n$ for all $t \in[a, b]$. Let $f_{K(t) ; E}^{\mu}(z), t \in[a, b]$, be the corresponding one-parameter family of $\mu$-polynomials. Then:

i) There exist $r-k-1$ continuous functions $\nu_{1}, \ldots, \nu_{r-k-1}:[a, b] \longrightarrow \mathbb{C}$ joining the $r-k-1$ non-zero roots of $f_{K(a) ; E}^{\mu}(z)$ and $r-k-1$ non-zero roots of $f_{K(b) ; E}^{\mu}(z)$, such that $\nu_{1}(t), \ldots, \nu_{r-k-1}(t)$ are $r-k-1$ of the $r-k$ non-zero roots of $f_{K(t) ; E}^{\mu}(z)$ for all $t \in[a, b]$.

ii) Moreover there exists another continuous function $\nu_{r-k}:(a, b] \longrightarrow \mathbb{C}$ such that $\nu_{r-k}(t)$ is the remaining root of $f_{K(t) ; E}^{\mu}(z)$ for all $t \in(a, b]$, verifying that $\lim _{t \rightarrow a^{+}} \nu_{r-k}(t)=0$.

Proof. Since $c(t)=\left(\begin{array}{l}n \\ r\end{array}\right) \mathrm{W}_{r}(K(t) ; E) m_{r}(\mu) \neq 0$ for all $t \in[a, b]$, the result is a direct consequence of Theorem 3.1 and the fact that the roots of a polynomial are continuous functions of the coefficients (see e.g. [14], page 3) applied to the polynomials

$$
\frac{f_{K(t) ; E}^{\mu}(z)}{c(t) z^{k}}=\frac{1}{c(t)} \sum_{i=0}^{r-k}\left(\begin{array}{c}
n \\
k+i
\end{array}\right) \mathrm{W}_{k+i}(K(t) ; E) m_{k+i}(\mu) z^{i},
$$

whose leading coefficients are 1 for all $t \in[a, b]$.

Proof of Theorem 1.2. By the homogeneity of the quermassintegrals we have that for $K, E \in \mathcal{K}^{n}$ and $\lambda>0, f_{\lambda K ; E}^{\mu}(\lambda z)=\lambda^{n} f_{K ; E}^{\mu}(z)$. Hence, if $\nu \in \mathcal{R}^{\mu}(n), \nu \neq 0$, then there exist $K, E \in \mathcal{K}^{n}$ such that $f_{K ; E}^{\mu}(\nu)=0$ and thus, for each $\lambda>0$, $0=f_{K ; E}^{\mu}(\nu)=f_{\lambda K ; E}^{\mu}(\lambda \nu) / \lambda^{n}$. Therefore $\lambda \nu \in \mathcal{R}^{\mu}(n)$ and so, $\mathcal{R}^{\mu}(n)$ is a cone.

In order to prove the convexity of $\mathcal{R}^{\mu}(n)$ it suffices to show that for any $\nu_{0} \in$ $\mathcal{R}^{\mu}(n)$ fixed, $\nu_{0} \neq 0$, the cone

$$
\mathcal{R}^{\mu}(n) \cap\left(\left\{z \in \mathbb{C}^{+} \backslash\{0\}: \arg z \geq \arg \nu_{0}\right\} \cup\{0\}\right)
$$


is convex. To this end, let $K, E \in \mathcal{K}^{n}$ be such that $f_{K ; E}^{\mu}\left(\nu_{0}\right)=0$. Without loss of generality we may assume that aff $E=\left\{x_{1}=\cdots=x_{n-r}=0\right\}$, where $r=\operatorname{dim} E$. Let $H_{i}=\left\{x_{n-r+1}=\cdots=x_{n-r+i}=0\right\}, i=1, \ldots, r-1$, be the $(n-i)$-dimensional coordinate plane containing (aff $E)^{\perp}$, and let $K_{i}=K \mid H_{i}$ be the orthogonal projection of $K$ onto $H_{i}, i=1, \ldots, r-1$, with $K_{0}=K$.

With this notation we will show, by finite induction on $j=r-i$, with $j=1, \ldots, r$, that all the points of $\mathcal{R}^{\mu}\left(K_{i} ; E\right)$ (cf. (3.1)) are roots of some $\mu$ polynomial, i.e., that $\mathcal{R}^{\mu}\left(K_{i} ; E\right) \subset \mathcal{R}^{\mu}(n)$. So $\mathcal{R}^{\mu}(K ; E) \subset \mathcal{R}^{\mu}(n)$, which will show the convexity of $\mathcal{R}^{\mu}(n) \cap\left(\left\{z \in \mathbb{C}^{+} \backslash\{0\}: \arg z \geq \arg \nu_{0}\right\} \cup\{0\}\right)$.

If $j=1$, then the polynomial $f_{K_{r-1} ; E}^{\mu}(z)$ reduces to

$$
\left[\left(\begin{array}{c}
n \\
r-1
\end{array}\right) \mathrm{W}_{r-1}\left(K_{r-1} ; E\right) m_{r-1}(\mu)+\left(\begin{array}{c}
n \\
r
\end{array}\right) \mathrm{W}_{r}\left(K_{r-1} ; E\right) m_{r}(\mu) z\right] z^{r-1}
$$

and so it has only a non-zero real root. Thus, $\mathbb{R}_{\leq 0}=\mathcal{R}^{\mu}\left(K_{r-1} ; E\right) \subset \mathcal{R}^{\mu}(n)$ and in particular, we have that $\mathcal{R}^{\mu}(n)$ contains the non-positive real axis.

Now we assume $1<j \leq r$ and that the result is true for $j-1$, i.e., we assume that $\mathcal{R}^{\mu}\left(K_{r-j+1} ; E\right) \subset \mathcal{R}^{\mu}(n)$. Notice that we can suppose the strict inclusion $\mathcal{R}^{\mu}\left(K_{r-j+1} ; E\right) \subsetneq \mathcal{R}^{\mu}\left(K_{r-j} ; E\right)$, otherwise we directly get the required result. For each $t \in[0,1]$, we consider the convex body

$$
K(t)=t K_{r-j+1}+(1-t) K_{r-j},
$$

and let $\nu_{j}$ be a root of $f_{K_{r-j} ; E}^{\mu}(z)$ such that $\arg \nu_{j}=\theta_{K_{r-j} ; E}^{\mu}$. The family of sets $K(t), t \in[0,1]$, provides a one-parameter family of $\mu$-polynomials $f_{K(t) ; E}^{\mu}(z)$ satisfying the conditions of Lemma 3.1, and hence there exists a continuous map $\nu:[0,1] \longrightarrow \mathbb{C}$ with $\nu(0)=\nu_{j}$ and $\nu(1)=\nu_{j-1}$ being a root of $f_{K_{r-j+1} ; E}^{\mu}(z)$, such that $\nu(t)$ is a root of $f_{K(t) ; E}^{\mu}(z)$ for all $t \in[0,1]$. Without loss of generality we may assume that $\nu_{j}$ is not the root which 'goes to zero'; otherwise, we can work with its conjugate $\bar{\nu}_{j}$.

Therefore, $f:[0,1] \longrightarrow(0,2 \pi), f(t)=\arg \nu(t)$, is a continuous function with $f(1)=\arg \nu_{j-1} \geq \theta_{K_{r-j+1} ; E}^{\mu}$ and $f(0)=\theta_{K_{r-j} ; E}^{\mu}$. Thus, using the intermediate value theorem, together with the fact that $\mathcal{R}^{\mu}(n)$ is a cone and the induction hypothesis, we may conclude that $\mathcal{R}^{\mu}\left(K_{r-j} ; E\right) \subset \mathcal{R}^{\mu}(n)$.

In [10], Kampf considered the already mentioned Wills type functionals associated to a signed measure $\rho$. We will show that in this case, the corresponding cone $\mathcal{R}^{\rho}(n)$ is not, in general, convex.

Proposition 3.1. There exist signed measures $\rho$ on $\mathbb{R}_{\geq 0}$ such that the cone $\mathcal{R}^{\rho}(n)$ is not convex.

Proof. Let $\rho$ be the signed measure on $\mathbb{R}_{\geq 0}$ given by

$$
\rho(\{0\})=\frac{7}{6}, \quad \rho(\{1\})=-\frac{1}{3}, \quad \rho(\{2\})=\frac{1}{6}, \quad \rho\left(\mathbb{R}_{\geq 0} \backslash\{0,1,2\}\right)=0 .
$$


Then the first four moments are given by

$$
\left(m_{0}(\rho), m_{1}(\rho), m_{2}(\rho), m_{3}(\rho)\right)^{\top}=A(7 / 6,-1 / 3,1 / 6)^{\top}=(1,0,1 / 3,1)^{\top},
$$

where $A$ is the matrix with entries $a_{11}=1$ and $a_{i j}=(j-1)^{(i-1)}$ for $(i, j) \neq(1,1)$, $1 \leq i \leq 4,1 \leq j \leq 3$. Hence, in dimension $n=3$, any $\rho$-polynomial takes the form $f_{K ; E}^{\rho}(z)=\mathrm{W}_{0}(K ; E)+\mathrm{W}_{2}(K ; E) z^{2}+\mathrm{W}_{3}(K ; E) z^{3}$.

On the one hand, if $K, E \in \mathcal{K}^{n}$ are convex bodies such that $\mathrm{W}_{0}(K ; E)=0$ and both $\mathrm{W}_{2}(K ; E), \mathrm{W}_{3}(K ; E) \neq 0$, i.e., with $1 \leq \operatorname{dim} K \leq 2$ and $\operatorname{dim} E=3$, then $f_{K ; E}^{\rho}(z)$ has only non-positive real roots.

On the other hand, if $K, E \in \mathcal{K}^{n}$ are such that $\mathrm{W}_{3}(K ; E)=0$ and both $\mathrm{W}_{0}(K ; E), \mathrm{W}_{2}(K ; E) \neq 0$, i.e., $\operatorname{dim} K=3$ and $\operatorname{dim} E=2$, then $f_{K ; E}^{\rho}(z)$ has imaginary pure complex roots.

Thus $\mathbb{R}_{\leq 0} \cup\{r \mathrm{i} \in \mathbb{C}: r \geq 0\} \subset \mathcal{R}^{\rho}(3) \cap\left\{z \in \mathbb{C}^{+}: \operatorname{Re} z \leq 0\right\}$. And moreover, it is easy to check that this inclusion is an equality: indeed, if there exist convex bodies $K, E \in \mathcal{K}^{n}$ such that

$$
f_{K ; E}^{\rho}(z)=\mathrm{W}_{3}(K ; E)(z+1+b \mathrm{i})(z+1-b \mathrm{i})(z+c)
$$

for some $b, c \geq 0$, with $\mathrm{W}_{3}(K ; E) \neq 0$, then we get, in particular, the relation $\mathrm{W}_{3}(K ; E)\left(b^{2}+2 c+1\right)=0$, which is a contradiction. This shows that the cone $\mathcal{R}^{\rho}(3)$ is not convex.

\subsection{Characterizing $\mu$-polynomials. Properties of $\mathcal{R}^{\mu}(n)$}

The main ingredient for most of the following proofs are the well-known inequalities

$$
\mathrm{W}_{i}(K ; E)^{2} \geq \mathrm{W}_{i-1}(K ; E) \mathrm{W}_{i+1}(K ; E), \quad 1 \leq i \leq n-1,
$$

particular cases of the Aleksandrov-Fenchel inequality (see e.g. Section 6.3 in [17]).

A sequence of real numbers $a_{0}, \ldots, a_{n} \geq 0$ is called ultra-logconcave if

$$
c_{i, n} a_{i}^{2} \geq a_{i-1} a_{i+1}, \quad \text { with } c_{i, n}=\frac{\left(\begin{array}{c}
n \\
i-1
\end{array}\right)\left(\begin{array}{c}
n \\
i+1
\end{array}\right)}{\left(\begin{array}{c}
n \\
i
\end{array}\right)^{2}},
$$

$1 \leq i \leq n-1$. In Lemma 2.1 and Corollary 2.1 of [8] (see also [18]), it is shown that this property allows to characterize Steiner polynomials. These results can be rewritten, with an analogous proof, to characterize $\mu$-polynomials for a given measure $\mu$. We include them here, without proof, for completeness.

For complex numbers $z_{1}, \ldots, z_{r} \in \mathbb{C}$ let

$$
\mathrm{s}_{i}\left(z_{1}, \ldots, z_{r}\right)=\sum_{\substack{J \subset\{1, \ldots, r\} \\ \# J=i}} \prod_{j \in J} z_{j}
$$

denote the $i$-th elementary symmetric function of $z_{1}, \ldots, z_{r}, 1 \leq i \leq r$. In addition we set $\mathrm{s}_{0}\left(z_{1}, \ldots, z_{r}\right)=1$. Moreover, let

$$
c_{i, n}^{\mu}=\frac{\left(\begin{array}{c}
n \\
i-1
\end{array}\right) m_{i-1}(\mu)\left(\begin{array}{c}
n \\
i+1
\end{array}\right) m_{i+1}(\mu)}{\left(\begin{array}{c}
n \\
i
\end{array}\right)^{2} m_{i}(\mu)^{2}} .
$$


Lemma 3.2. A real polynomial $\sum_{i=0}^{n} a_{i} z^{i}, a_{i} \geq 0$, is a $\mu$-polynomial $f_{K ; E}^{\mu}(z)$ for a measure $\mu$ on $\mathbb{R}_{\geq 0}$ and a pair $K, E \in \mathcal{K}^{n}$, with $\operatorname{dim} E=r, \operatorname{dim} K=n-k$, $\operatorname{dim}(K+E)=n$, if and only if $a_{i}>0$ for all $k \leq i \leq r$, and $a_{i}=0$ otherwise, and the sequence $\left(a_{i} / m_{i}(\mu)\right)_{i=0}^{n}$ is ultra-logconcave.

Moreover, $\nu_{1}, \nu_{2}, \ldots, \nu_{r} \in \mathbb{C}$ are the roots of the $\mu$-polynomial $f_{K ; E}^{\mu}(z)$ of degree $r \leq n, \operatorname{dim} E=r, \operatorname{dim} K=n-k, \operatorname{dim}(K+E)=n$, if and only if

i) $(-1)^{i} \mathrm{~s}_{i}\left(\nu_{1}, \ldots, \nu_{r}\right)>0, \quad 0 \leq i \leq r-k$, $\mathrm{s}_{i}\left(\nu_{1}, \ldots, \nu_{r}\right)=0, \quad r-k+1 \leq i \leq r$,

ii) $c_{r-i, n}^{\mu} \mathrm{s}_{i}\left(\nu_{1}, \ldots, \nu_{r}\right)^{2} \geq \mathrm{s}_{i-1}\left(\nu_{1}, \ldots, \nu_{r}\right) \mathrm{s}_{i+1}\left(\nu_{1}, \ldots, \nu_{r}\right), \quad 1 \leq i \leq r-1$.

We notice that an analogous result to Lemma 3.2 can be stated for $\mathbf{m}$-polynomials $f_{K ; E}^{\mathbf{m}}(z)$.

Regarding the topology (closeness) of the cone $\mathcal{R}^{\mu}(n)$, the proof of Theorem 1.3 is similar to the corresponding one for Steiner polynomials (see Theorem 1.2 in [8]). We briefly sketch it here, pointing out the slight differences, for completeness.

Proof of Theorem 1.3. Let $\nu \in \operatorname{bd} \mathcal{R}^{\mu}(n)$. Since $\mathbb{R}_{\leq 0} \subset \mathcal{R}^{\mu}(n)$ (see Theorem 1.2), we assume that $\nu \notin \mathbb{R}$. Let $\left(\nu_{j}\right)_{j \in \mathbb{N}} \subsetneq \operatorname{int} \mathcal{R}^{\mu}(n)$ be such that $\lim _{j \rightarrow \infty} \nu_{j}=\nu$. For each $j \in \mathbb{N}$, since $\nu_{j} \in \mathcal{R}^{\mu}(n)$, then there exist $K_{j}, E_{j} \in \mathcal{K}^{n}, \operatorname{dim}\left(K_{j}+E_{j}\right)=n$, with $f_{K_{j} ; E_{j}}^{\mu}\left(\nu_{j}\right)=0$.

We notice that among all pairs of convex bodies with $\nu_{j}$ as a root of the corresponding $\mu$-polynomial, we can always choose $K_{j}, E_{j}$ such that $f_{K_{j} ; E_{j}}^{\mu}(1)=1$; otherwise, since $f_{K_{j} ; E_{j}}^{\mu}(1)>0$, we consider the convex bodies $K_{j}^{\prime}=f_{K_{j} ; E_{j}}^{\mu}(1)^{-1 / n} K_{j}$, $E_{j}^{\prime}=f_{K_{j} ; E_{j}}^{\mu}(1)^{-1 / n} E_{j}$, for which it clearly holds $f_{K_{j}^{\prime} ; E_{j}^{\prime}}^{\mu}\left(\nu_{j}\right)=0$ and $f_{K_{j}^{\prime} ; E_{j}^{\prime}}^{\mu}(1)=1$.

Since $f_{K_{j} ; E_{j}}^{\mu}(1)=\sum_{i=0}^{n}\left(\begin{array}{c}n \\ i\end{array}\right) \mathrm{W}_{i}\left(K_{j} ; E_{j}\right) m_{i}(\mu)=1$, then all quermassintegrals $\mathrm{W}_{i}\left(K_{j} ; E_{j}\right) \in\left[0,1 / \min _{0 \leq l \leq n}\left\{m_{l}(\mu)\right\}\right], i=0, \ldots, n$, and not all of them are zero. The proof finishes with a (sequential) compactness argument (see the proof of Theorem 1.2 in [8]), using the characterization of $\mu$-polynomials via ultra-logconcave sequences (see Lemma 3.2).

The monotonicity $\mathcal{R}^{\mu}(n) \subset \mathcal{R}^{\mu}(n+1)$ is easy to prove. Let $\nu \in \mathcal{R}^{\mu}(n)$ and let $K, E \in \mathcal{K}^{n}, \operatorname{dim}(K+E)=n$, be such that $f_{K ; E}^{\mu}(\nu)=0$. Embedding $K$ canonically into the hyperplane $\left\{\mathrm{e}_{n+1}\right\}^{\perp} \subsetneq \mathbb{R}^{n+1}$ ( $\mathrm{e}_{i}$ denotes the $i$-th canonical unit vector), let $K^{\prime}=K \times \operatorname{conv}\left\{0, \mathrm{e}_{n+1}\right\}$ be the prism over $K$ of height 1 in the direction $\mathrm{e}_{n+1}$. Then $\operatorname{vol}_{n+1}\left(K^{\prime}+\lambda E\right)=\operatorname{vol}_{n}(K+\lambda E)$ for all $\lambda \geq 0$, and thus (cf. (1.1)),

$$
\begin{aligned}
\left(\begin{array}{c}
n+1 \\
i
\end{array}\right) \mathrm{W}_{i}^{(n+1)}\left(K^{\prime} ; E\right) & =\left(\begin{array}{c}
n \\
i
\end{array}\right) \mathrm{W}_{i}^{(n)}(K ; E), \quad i=0, \ldots, n, \\
\mathrm{~W}_{n+1}^{(n+1)}\left(K^{\prime} ; E\right) & =0
\end{aligned}
$$

here $\mathrm{W}_{i}^{(j)}$ denotes the $i$-th quermassintegral in $\mathbb{R}^{j}$. Multiplying the above identities by $m_{i}(\mu)$ and $m_{n+1}(\mu)$ respectively, we obtain $f_{K^{\prime} ; E}^{\mu}(z)=f_{K ; E}^{\mu}(z)$, and thus $f_{K^{\prime} ; E}^{\mu}(\nu)=0$. Hence $\nu \in \mathcal{R}^{\mu}(n+1)$. 
Next we show that the inclusion $\mathcal{R}^{\mu}(n) \subset \mathcal{R}^{\mu}(n+1)$ is strict (Theorem 1.4). The proof is similar to the one of Theorem 1.3 in [8]. We again sketch it here, briefly pointing out the differences.

Proof of Theorem 1.4. In order to show that the above inclusion is indeed strict, let $\nu \in \operatorname{bd} \mathcal{R}^{\mu}(n) \backslash \mathbb{R}_{\leq 0}$; otherwise the assertion is trivially true (see e.g. Theorem 1.5 for $n=2$, and Theorem 1.2 in [7]). Since $\mathcal{R}^{\mu}(n)$ is closed (Theorem 1.3), $\nu$ is a root of some $\mu$-polynomial $f_{K ; E}^{\mu}(z)$ of degree $r \leq n$, with $K, E \in \mathcal{K}^{n}, \operatorname{dim} E=r$, $\operatorname{dim} K=n-k, \operatorname{dim}(K+E)=n$. Let $\bar{\nu}, \nu_{3}, \ldots, \nu_{r}$ be the remaining roots of the polynomial.

Then, we have to see that there exists $\varepsilon>0$ small enough such that, for any $z \in \mathbb{C},|z|=1$, the $r$ numbers $\nu+\varepsilon z, \bar{\nu}+\varepsilon \bar{z}, \nu_{3}, \ldots, \nu_{r}$ are roots of a suitable $\mu$-polynomial $f_{K^{\prime} ; E^{\prime}}^{\mu}(z), K^{\prime}, E^{\prime} \in \mathcal{K}^{n+1}$ with $\operatorname{dim} K^{\prime}=n-k+1, \operatorname{dim} E^{\prime}=r$ and $\operatorname{dim}\left(K^{\prime}+E^{\prime}\right)=n+1$, i.e., the 'sign conditions' i) and the 'quadratic conditions' ii) in Lemma 3.2 are properly verified, namely,

i') $(-1)^{i} \mathrm{~s}_{i}\left(\nu+\varepsilon z, \bar{\nu}+\varepsilon \bar{z}, \nu_{3}, \ldots, \nu_{r}\right)>0, \quad 0 \leq i \leq r-k$,

$$
\mathrm{s}_{i}\left(\nu+\varepsilon z, \bar{\nu}+\varepsilon \bar{z}, \nu_{3}, \ldots, \nu_{r}\right)=0, \quad r-k+1 \leq i \leq r,
$$

and

$$
\text { ii') } \begin{aligned}
c_{r-i, n+1}^{\mu} & \mathrm{s}_{i}\left(\nu+\varepsilon z, \bar{\nu}+\varepsilon \bar{z}, \nu_{3}, \ldots, \nu_{r}\right)^{2} \\
& \geq \mathrm{s}_{i-1}\left(\nu+\varepsilon z, \bar{\nu}+\varepsilon \bar{z}, \nu_{3}, \ldots, \nu_{r}\right) \mathrm{s}_{i+1}\left(\nu+\varepsilon z, \bar{\nu}+\varepsilon \bar{z}, \nu_{3}, \ldots, \nu_{r}\right),
\end{aligned}
$$

for $1 \leq i \leq r-1$. Since conditions i) and ii) of Lemma 3.2 hold for the sequence of roots $\nu, \bar{\nu}, \nu_{3}, \ldots, \nu_{r}$, and since

$$
\begin{aligned}
c_{r-i, n+1}^{\mu} & =\frac{\left(\begin{array}{c}
n+1 \\
r-i-1
\end{array}\right)\left(\begin{array}{c}
n+1 \\
r-i+1
\end{array}\right)}{\left(\begin{array}{c}
n+1 \\
r-i
\end{array}\right)^{2}} \frac{m_{r-i-1}(\mu) m_{r-i+1}(\mu)}{m_{r-i}(\mu)^{2}} \\
> & \frac{\left(\begin{array}{c}
n \\
r-i-1
\end{array}\right)\left(\begin{array}{c}
n \\
r-i+1
\end{array}\right)}{\left(\begin{array}{c}
n \\
r-i
\end{array}\right)^{2}} \frac{m_{r-i-1}(\mu) m_{r-i+1}(\mu)}{m_{r-i}(\mu)^{2}}=c_{r-i, n}^{\mu}
\end{aligned}
$$

for all $1 \leq i \leq r-1$, the proof of the above facts i') and ii') is just a consequence of the continuity of the elementary symmetric functions (see the proof of Theorem 1.3 in $[8])$.

The following proposition is also analogous to the corresponding one for Steiner polynomials (see Corollary 1.1 in [8]).

Proposition 3.2. For $n \geq 3$ and a given measure $\mu$ on $\mathbb{R}_{\geq 0}$, let $K, E \in \mathcal{K}^{n}$ be such that the $\mu$-polynomial $f_{K ; E}^{\mu}(z)$ has a root lying on $\operatorname{bd} \mathcal{R}^{\mu}(n) \backslash \mathbb{R}_{\leq 0}$. Then $K, E$ are extremal sets for at least one Aleksandrov-Fenchel inequality.

Proof. For $\nu \in \operatorname{bd} \mathcal{R}^{\mu}(n) \backslash \mathbb{R}_{\leq 0}$, let $K, E \in \mathcal{K}^{n}$, with $\operatorname{dim} E=r$, be such that $f_{K ; E}^{\mu}(\nu)=0$, and let $\bar{\nu}, \nu_{3}, \ldots, \nu_{r}$ be the remaining roots of $f_{K ; E}^{\mu}(z)$. If $K, E$ are not extremal sets in any Aleksandrov-Fenchel inequality, i.e., if we have strict inequalities in (3.2), then $r \geq n-1$ and for all $1 \leq i \leq r-1$ it holds

$$
c_{r-i, n}^{\mu} \mathrm{s}_{i}\left(\nu, \bar{\nu}, \nu_{3}, \ldots, \nu_{r}\right)^{2}>\mathrm{s}_{i-1}\left(\nu, \bar{\nu}, \nu_{3}, \ldots, \nu_{r}\right) \mathrm{s}_{i+1}\left(\nu, \bar{\nu}, \nu_{3}, \ldots, \nu_{r}\right) .
$$


Again, by the continuity of the elementary symmetric functions, for $\varepsilon>0$ small enough, the numbers $\nu+\varepsilon z, \bar{\nu}+\varepsilon \bar{z}, \nu_{3}, \ldots, \nu_{n}$ are roots of a polynomial with real coefficients, satisfying conditions i) and ii) in Lemma 3.2 for any $z \in \mathbb{C}$ with $|z|=1$. Thus $\{\nu+\varepsilon z:|z|=1\} \subsetneq \mathcal{R}^{\mu}(n)$, a contradiction.

\subsection{The cone of roots for a fixed gauge body}

Let $E \in \mathcal{K}^{n}$ be a fixed gauge body. If we define

$$
\mathcal{R}^{\mu}(n ; E)=\left\{z \in \mathbb{C}^{+}: f_{K ; E}^{\mu}(z)=0 \text { for } K \in \mathcal{K}^{n}, \operatorname{dim}(K+E)=n\right\},
$$

then the proof of Theorem 1.2 also says that $\mathcal{R}^{\mu}(n ; E)$ is a convex cone, containing the non-positive real axis $\mathbb{R}_{\leq 0}$. However, other properties of $\mathcal{R}^{\mu}(n)$ cannot be extended to $\mathcal{R}^{\mu}(n ; E)$. For instance, $\mathcal{R}^{\mu}(n ; E)$ is, in general, not closed (see e.g. Theorem 1.2 in [7]). Here we give a sufficient condition for $\nu \in \operatorname{bd} \mathcal{R}^{\mu}(n ; E) \backslash \mathbb{R}_{\leq 0}$ to lie in $\mathcal{R}^{\mu}(n ; E)$.

Let $\mathrm{R}(K)=\min \left\{R>0: \exists x \in \mathbb{R}^{n}\right.$ with $\left.K \subset x+R B_{n}\right\}$ denote the circumradius of the convex body $K$. The (unique) point $x$ such that $K \subset x+\mathrm{R}(K) B_{n}$ is called its circumcenter.

Proposition 3.3. Let $\nu \in \operatorname{bd} \mathcal{R}^{\mu}(n ; E) \backslash \mathbb{R}_{\leq 0}$. Let $\left(\nu_{j}\right)_{j \in \mathbb{N}} \subsetneq \operatorname{int} \mathcal{R}^{\mu}(n ; E)$ with $\lim _{j \rightarrow \infty} \nu_{j}=\nu$ and, for each $j \in \mathbb{N}$, let $K_{j} \in \mathcal{K}^{n}, \operatorname{dim}\left(K_{j}+E\right)=n$, be such that $f_{K_{j} ; E}^{\mu}\left(\nu_{j}\right)=0$. If there exists a subsequence $\left(K_{j_{m}}\right)_{m \in \mathbb{N}} \subset\left(K_{j}\right)_{j \in \mathbb{N}}$ with $\operatorname{dim} K_{j_{m}}=$ $n-k$ for all $m \in \mathbb{N}$, such that

$$
\lim _{m \rightarrow \infty} \frac{\mathrm{W}_{k}\left(K_{j_{m}} ; E\right)}{\mathrm{R}\left(K_{j_{m}}\right)^{n-k}} \neq 0,
$$

then $\nu \in \mathcal{R}^{\mu}(n ; E)$.

Proof. Without loss of generality, we may suppose that $\operatorname{dim} K_{j}=n-k$ for all $j \in \mathbb{N}$ and thus $\mathrm{W}_{k}\left(K_{j} ; E\right) \neq 0$.

For each $j \in \mathbb{N}$, let $\widetilde{K}_{j}=\mathrm{R}\left(K_{j}\right)^{-1} K_{j}$ and $\widetilde{\nu}_{j}=\mathrm{R}\left(K_{j}\right)^{-1} \nu_{j}$, which is a root of $f_{\widetilde{K}_{j} ; E}^{\mu}(z)$. Since quermassintegrals are translation invariant (and thus the corresponding $\mu$-polynomial), it is not restrictive to assume that the origin 0 is the circumcenter of all $K_{j}$, and so, of $\widetilde{K}_{j}$. Then, $\widetilde{K}_{j} \subset B_{n}$ for all $j \in \mathbb{N}$, and Blaschke's selection theorem (see e.g. Theorem 1.8.6 in [17]) ensures the existence of a subsequence of $\left(\widetilde{K}_{j}\right)_{j \in \mathbb{N}}$ converging to a convex body $K \in \mathcal{K}^{n}$; without loss of generality we may assume that $\lim _{j \rightarrow \infty} \widetilde{K}_{j}=K$. Then, by the continuity of the quermassintegrals, each coefficient $\left(\begin{array}{c}n \\ i\end{array}\right) \mathrm{W}_{i}\left(\widetilde{K}_{j} ; E\right) m_{i}(\mu)$ of the polynomial $f_{\widetilde{K}_{j} ; E}^{\mu}(z)$ converges to the corresponding coefficient $\left(\begin{array}{c}n \\ i\end{array}\right) \mathrm{W}_{i}(K ; E) m_{i}(\mu)$ of $f_{K ; E}^{\mu}(z)$, and moreover,

$$
\begin{aligned}
\mathrm{W}_{k}(K ; E) & =\mathrm{W}_{k}\left(\lim _{j \rightarrow \infty} \tilde{K}_{j} ; E\right)=\lim _{j \rightarrow \infty} \mathrm{W}_{k}\left(\widetilde{K}_{j} ; E\right) \\
& =\lim _{j \rightarrow \infty} \mathrm{W}_{k}\left(\frac{1}{\mathrm{R}\left(K_{j}\right)} K_{j} ; E\right)=\lim _{j \rightarrow \infty} \frac{\mathrm{W}_{k}\left(K_{j} ; E\right)}{\mathrm{R}\left(K_{j}\right)^{n-k}} \neq 0 .
\end{aligned}
$$


Hence, from the fact that the roots of a polynomial are continuous functions of the coefficients of the polynomial (see e.g. [14], page 3), and since $f_{\widetilde{K}_{j} ; E}^{\mu}(z)$ has degree $r=\operatorname{dim} E$ for all $j \in \mathbb{N}$, we get that there exist $r-k$ sequences of numbers $\left(\nu_{j}^{1}\right)_{j \in \mathbb{N}}, \ldots,\left(\nu_{j}^{r-k}\right)_{j \in \mathbb{N}}$ such that $\nu_{j}^{1}, \ldots, \nu_{j}^{r-k}$ are the $r-k$ non-zero roots of $f_{\widetilde{K}_{j} ; E}^{\mu}(z)$ for all $j \in \mathbb{N}$, and with $\nu^{i}=\lim _{j \rightarrow \infty} \nu_{j}^{i}, i=1, \ldots, r-k$, being the nonzero roots of $f_{K ; E}^{\mu}(z)$. Now, since $\widetilde{\nu}_{j}$ was a non-zero root of $f_{\widetilde{K}_{j} ; E}^{\mu}(z)$ for each $j$, taking subsequences if necessary, we may assume that $\left(\widetilde{\nu}_{j}\right)_{j \in \mathbb{N}}$ converges to some root of $f_{K ; E}^{\mu}(z)$, say $\nu^{1}$. Then

$$
\frac{\nu}{\nu^{1}}=\frac{\lim _{j \rightarrow \infty} \nu_{j}}{\lim _{j \rightarrow \infty} \widetilde{\nu}_{j}}=\lim _{j \rightarrow \infty} \frac{\nu_{j}}{\widetilde{\nu}_{j}}=\lim _{j \rightarrow \infty} \mathrm{R}\left(K_{j}\right) \in \mathbb{R}_{>0},
$$

which implies that $f_{\left(\nu / \nu^{1}\right) K ; E}^{\mu}(\nu)=0$, as required.

\section{The smallest and largest cones of roots of $\mu$-polynomials}

Let $C_{n}$ denote the $n$-dimensional regular cube of edge-length 1 . Then, the Aleksandrov-Fenchel inequalities (3.2) for $\mathrm{W}_{i}\left(C_{n} ; B_{n}\right)=\kappa_{i}$ and any value of the dimension yield the inequalities

$$
m_{i}(g)^{2} \leq m_{i+1}(g) m_{i-1}(g), \quad i=1,2, \ldots,
$$

for the moments $m_{i}(g)=1 / \kappa_{i}$ of the measure $g$ associated to $G(t)=e^{-\pi t^{2}}$.

Moreover, if we had equality in one of the above inequalities for an $i \geq 1$, i.e., if $\mathrm{W}_{i}\left(C_{n} ; B_{n}\right)^{2}=\mathrm{W}_{i-1}\left(C_{n} ; B_{n}\right) \mathrm{W}_{i+1}\left(C_{n} ; B_{n}\right)$, then the known equality case in Aleksandrov-Fenchel inequality for centrally symmetric convex bodies would lead to a contradiction (it would imply that $C_{n}$ is a so called $(n-i-1)$-tangential body of a ball with $i \neq 0$, which is not true; see Theorem 6.6.19 in [17]). Thus, inequalities (4.1) are strict.

This fact can be extended to the moments of (almost) any measure; it will be shown and used in the proof of Theorem 1.5.

Proof of Theorem 1.5. Let $\gamma \in \mathcal{R}(n)$. Then there exist convex bodies $K, E \in \mathcal{K}^{n}$ such that $f_{K ; E}(\gamma)=\sum_{i=0}^{n}\left(\begin{array}{c}n \\ i\end{array}\right) \mathrm{W}_{i}(K ; E) \gamma^{i}=0$.

Moreover, for any measure $\mu$ on $\mathbb{R}_{\geq 0}$, the Cauchy-Schwarz inequality yields

$$
\begin{aligned}
m_{i}(\mu)^{2} & =\left(\int_{0}^{\infty} t^{i} \mathrm{~d} \mu(t)\right)^{2}=\left(\int_{0}^{\infty} t^{(i+1) / 2} t^{(i-1) / 2} \mathrm{~d} \mu(t)\right)^{2} \\
& \leq \int_{0}^{\infty} t^{i+1} \mathrm{~d} \mu(t) \int_{0}^{\infty} t^{i-1} \mathrm{~d} \mu(t)=m_{i+1}(\mu) m_{i-1}(\mu),
\end{aligned}
$$

i.e., $1 / m_{i}(\mu), i=0, \ldots, n$, satisfy the Aleksandrov-Fenchel inequalities (3.2). Then, we get that the sequence of positive numbers $\left(\begin{array}{c}n \\ i\end{array}\right) \mathrm{W}_{i}(K ; E) / m_{i}(\mu)$ is ultralogconcave, and Lemma 3.2 ensures that the Steiner polynomial $f_{K ; E}(z)$ is also a $\mu$-polynomial for some convex bodies $K^{\prime}, E^{\prime} \in \mathcal{K}^{n}$. Therefore, $\gamma \in \mathcal{R}^{\mu}(n)$, as required. 
In order to prove the last assertion, we notice that equality in the CauchySchwarz inequality (4.2) holds if and only if

$$
t^{\frac{i+1}{2}}-\frac{m_{i}(\mu)}{m_{i-1}(\mu)} t^{\frac{i-1}{2}} \equiv 0
$$

almost everywhere or, in other words, if and only if

$$
\mu\left(\mathbb{R}_{\geq 0} \backslash\left\{0, m_{i}(\mu) / m_{i-1}(\mu)\right\}\right)=0, \quad i \geq 1 .
$$

Therefore, if a measure $\mu$ verifies the condition of the theorem, inequalities (3.2) strictly hold for the values $1 / m_{i}(\mu), i=1, \ldots, n-1$, which implies that $c_{r-i, n}^{\mu}>$ $c_{r-i, n}$; here, as usual, $r=\operatorname{dim} E=\operatorname{dim} E^{\prime}$. Hence, denoting by $\gamma_{2}, \ldots, \gamma_{r}$ the remaining roots of $f_{K ; E}(z)=f_{K^{\prime} ; E^{\prime}}^{\mu}(z)$, we get

$$
\begin{aligned}
c_{r-i, n}^{\mu} \mathrm{s}_{i}\left(\gamma, \gamma_{2}, \ldots, \gamma_{r}\right)^{2} & >c_{r-i, n} \mathrm{~s}_{i}\left(\gamma, \gamma_{2}, \ldots, \gamma_{r}\right)^{2} \\
& \geq \mathrm{s}_{i-1}\left(\gamma, \gamma_{2}, \ldots, \gamma_{r}\right) \mathrm{s}_{i+1}\left(\gamma, \gamma_{2}, \ldots, \gamma_{r}\right),
\end{aligned}
$$

which implies, if $n \geq 3$, that $\gamma \in \operatorname{int} \mathcal{R}^{\mu}(n)$ (see the proof of Proposition 3.2). Therefore, $\mathcal{R}(n) \subsetneq \mathcal{R}^{\mu}(n)$ when $n \geq 3$. For $n=2$, we just notice that the discriminant of $f_{K ; E}^{\mu}(z)$ is

$$
\Delta=m_{1}(\mu)^{2}\left(4 \mathrm{~W}_{1}(K ; E)^{2}-4 \operatorname{vol}(K) \operatorname{vol}(E) \frac{m_{0}(\mu) m_{2}(\mu)}{m_{1}(\mu)^{2}}\right),
$$

and thus, if $K=E=B_{2}$ it holds $\Delta<0$. Hence $\mathcal{R}(2)=\mathbb{R}_{\leq 0} \subsetneq \mathcal{R}^{\mu}(2)$.

As a consequence of Theorem 1.1 and the known results for the roots of the Steiner polynomial (see Proposition 1.3 and Theorem 1.4 in [8]), we directly get Corollary 1.1.

Remark 4.1. It is known that, for any $-a \in \mathbb{R}_{<0}, a>0$, there exist convex bodies $K, E \in \mathcal{K}^{n}$ such that $-a$ is an $n$-fold root of the Steiner polynomial $f_{K ; E}(z)$ (see e.g. Proposition 2.3 in [8]). This property remains true for any $\mu$-polynomial. Indeed, since $1 / m_{i}(\mu), i=0, \ldots, n$, satisfy the Aleksandrov-Fenchel inequalities (3.2) (see (4.2)), then Lemma 3.2 ensures that

$$
\sum_{i=0}^{n}\left(\begin{array}{l}
n \\
i
\end{array}\right) z^{i}=(z+1)^{n}=f_{K^{\prime} ; E^{\prime}}^{\mu}(z)
$$

is a $\mu$-polynomial for two convex bodies $K^{\prime}, E^{\prime} \in \mathcal{K}^{n}$, i.e., -1 is an $n$-fold root of $f_{K^{\prime} ; E^{\prime}}^{\mu}(z)$; for the real number $-a$, it suffices to consider $f_{K^{\prime} ;(1 / a) E^{\prime}}^{\mu}(z)$.

Several of the above properties for the cone of roots of $\mu$-polynomials (convexity, closeness, monotonicity in the dimension, etc.) remain true for general m-polynomials (see (1.4)), independently the numbers $m_{i}$ are moments of a measure on $\mathbb{R}_{\geq 0}$ or not; in fact, we have only needed that $m_{i}(\mu)>0$ for all $i \geq 0$. However, the properties collected in Theorem 1.5 and Corollary 1.1 are not true for arbitrary $\mathbf{m}$-polynomials, as the following example shows. 
Remark 4.2. Let $\mathbf{m}=\left(e^{-i(i+1) / 2}\right)_{i \in \mathbb{N}}$ and we consider the $\mathbf{m}$-polynomial $f_{K ; E}^{\mathbf{m}}(z)$ for any $K, E \in \mathcal{K}^{n}$. On the one hand, it is easy to check that

$$
\frac{m_{i-1}}{m_{i}} \leq \beta \frac{m_{i+1}}{m_{i+2}} \quad \text { for all } i=1,2, \ldots,
$$

where $\beta \approx 0.4655$ is the only real solution of the equation $z(z+1)^{2}=1$. On the other hand, it is known (see Theorem 3 in [16] and Theorem 1 in [11]) that

$$
\begin{aligned}
& f(z)=\sum_{i=0}^{n} a_{i} z^{i} \text {, with } a_{i}>0 \text { for } i=0, \ldots, n \text {, is stable (i.e., all its } \\
& \text { roots have negative real part) if } a_{i-1} a_{i+2} \leq \beta a_{i} a_{i+1} .
\end{aligned}
$$

Then, using (3.2) and (4.3) we get that $f_{K ; E}^{\mathrm{m}}(z)$ fulfills the above stability criterion (4.4) for any pair of convex bodies $K, E \in \mathcal{K}^{n}$ and any value of the dimension $n$. Therefore, Corollary 1.1 does not hold.

Thus, Theorem 1.5 and Corollary 1.1 provide necessary 'geometric' conditions for a sequence of positive numbers $\left\{m_{i}: i=0,1, \ldots\right\}$ to be the moments of a measure on $\mathbb{R}_{\geq 0}$.

So we already know that the 'smallest' cone of roots of $\mu$-polynomials is the one given by the Steiner polynomial. Next we deal with the 'largest' cone of roots of $\mu$-polynomials, i.e., we prove Theorem 1.6.

We observe that, in the proof of Theorem 1.5, the main tool in order to get the desired inclusion was the inequality $m_{i+1}(\mu) m_{i-1}(\mu) \geq m_{i}(\mu)^{2}$ for all $i \geq 1$. So, for a 'reverse' inclusion we would need that

$$
m_{i+1}(\mu) m_{i-1}(\mu) \leq c_{i} m_{i}(\mu)^{2}, \quad i \geq 1,
$$

for a suitable sequence $\left(c_{i}\right)_{i \in \mathbb{N}}$. Theorem 1.6 determines such a sequence, and thus the corresponding inclusion when working with log-concave measures, i.e., measures $\mu$ of the form $\mu(A)=\int_{A} f(t) \mathrm{d} t$, where $f$ is a log-concave function.

Proof of Theorem 1.6. We consider the real functions $f, g:[0, \infty) \rightarrow[0, \infty)$ given by $f(t)=t^{i-1}, g(t)=t^{i+1}$, and let

$$
h(t)=\sup \left\{f(x)^{1 / 2} g(y)^{1 / 2}: t=\frac{x+y}{2}, x, y \in[0, \infty)\right\} .
$$

It clearly holds $h((x+y) / 2) \geq f(x)^{1 / 2} g(y)^{1 / 2}$ for all $x, y \in[0, \infty)$ and hence, since $\mu$ is log-concave, by the Prékopa-Leindler inequality (see e.g. Theorem 8.14 in [4]),

$$
\int_{0}^{\infty} h(t) \mathrm{d} \mu(t) \geq\left(\int_{0}^{\infty} t^{i-1} \mathrm{~d} \mu(t)\right)^{1 / 2}\left(\int_{0}^{\infty} t^{i+1} \mathrm{~d} \mu(t)\right)^{1 / 2},
$$

i.e., we get

$$
\left(\int_{0}^{\infty} h(t) \mathrm{d} \mu(t)\right)^{2} \geq m_{i-1}(\mu) m_{i+1}(\mu)
$$


So, now we deal with the left-hand side in the above inequality. Taking into account the definition of $h$, we maximize, for each $t \geq 0$, the function $F_{t}:[-t, t] \rightarrow[0, \infty)$ given by

$$
F_{t}(s)=(t-s)^{(i-1) / 2}(t+s)^{(i+1) / 2} .
$$

Its first derivative $\left(F_{t}\right)^{\prime}(s)=\left(t^{2}-s^{2}\right)^{(i-1) / 2-1}\left(-i s^{2}+(1-i) t s+t^{2}\right)$ has only one root in $(-t, t)$, namely, $t / i$, which gives in fact the maximum of the function. Therefore, we have

$$
h(t)=F_{t}\left(\frac{t}{i}\right)= \begin{cases}t^{i} \frac{(i-1)^{(i-1) / 2}(i+1)^{(i+1) / 2}}{i^{i}} & \text { for } i>1, \\ 2 t & \text { for } i=1 .\end{cases}
$$

So, denoting by

$$
c_{i}=\frac{(i-1)^{(i-1)}(i+1)^{(i+1)}}{i^{2 i}} \text { for all } i>1, \quad c_{1}=4,
$$

we get that

$$
\left(\int_{0}^{\infty} h(t) \mathrm{d} \mu(t)\right)^{2}=\left(\int_{0}^{\infty} c_{i}^{1 / 2} t^{i} \mathrm{~d} \mu(t)\right)^{2}=c_{i} m_{i}(\mu)^{2},
$$

and thus (4.5) yields

$$
c_{i} m_{i}(\mu)^{2} \geq m_{i-1}(\mu) m_{i+1}(\mu)
$$

Now we can prove the result. Let $\nu \in \mathcal{R}^{\mu}(n)$. Then there exist convex bodies $K, E \in \mathcal{K}^{n}$ such that $f_{K ; E}^{\mu}(\nu)=\sum_{i=0}^{n}\left(\begin{array}{c}n \\ i\end{array}\right) \mathrm{W}_{i}(K ; E) m_{i}(\mu) \nu^{i}=0$.

Let $\boldsymbol{\omega}=\left(\omega_{i}\right)_{i \in \mathbb{N}}=\left(1,1,2^{2}, \ldots, i^{i}, \ldots\right)$. By (3.2) and (4.6), we easily get that the sequence of positive numbers $\left(\begin{array}{c}n \\ i\end{array}\right) \mathrm{W}_{i}(K ; E) m_{i}(\mu) / \omega_{i}, i=0, \ldots, n$, is ultralogconcave, and hence, $f_{K ; E}^{\mu}(z)$ is also an $\boldsymbol{\omega}$-polynomial $f_{K^{\prime} ; E^{\prime}}^{\boldsymbol{\omega}}(z)$ for some convex bodies $K^{\prime}, E^{\prime} \in \mathcal{K}^{n}$ (cf. Lemma 3.2). Thus, $f_{K^{\prime} ; E^{\prime}}^{\omega}(\nu)=0$, i.e., $\nu \in \mathcal{R}^{\omega}(n)$, as required.

\section{On the stability of generalized Wills $\mu$-polynomials}

We conclude the paper considering the stability of Wills $\mu$-polynomials, i.e., we study the inclusion

$$
\mathcal{R}^{\mu}(n) \subset\left\{z \in \mathbb{C}^{+}: \operatorname{Re} z<0\right\} \cup\{0\},
$$

property that we call, following the notation in [8], 'weak' stability.

Of course, it is not possible to state a general characterization result for $\mu$ polynomials for any measure $\mu$. So, it is natural to consider particularly prominent polynomials of this type, which, at the end, will provide information on the stability of any $\mu$-polynomial. 
Theorem 1.5 shows that the 'smallest' cone of roots of Wills $\mu$-polynomials is the one given by the Steiner polynomial, and it is known that Steiner polynomials are weakly stable if and only if $n \leq 9$ (see Proposition 1.3 in [8]). Therefore, we can state the following result:

Corollary 5.1. If $n \geq 10$ then, for any measure $\mu$ on $\mathbb{R}_{\geq 0}, \mu$-polynomials are not weakly stable, i.e., $\left\{z \in \mathbb{C}^{+}: \operatorname{Re} z \leq 0\right\} \subset \mathcal{R}^{\mu}(n)$.

Thus, we wonder for the stability of those polynomials which determine the 'largest' cone of roots containing $\mathcal{R}^{\mu}(n)$ for any log-concave measure $\mu$ on $\mathbb{R}_{\geq 0}$, i.e., the $\boldsymbol{\omega}$-polynomials (Theorem 1.6). We prove the following result.

Proposition 5.1. $\boldsymbol{\omega}$-polynomials are weakly stable if and only if $n \leq 3$.

Proof. First we notice that the stability criterion (4.4) cannot be applied to $f_{K ; E}^{\omega}(z)$ when $n=3$ because it is, in general, not fulfilled. Thus, we assume that $\boldsymbol{\omega}$ polynomials are not weakly stable in dimension $n=3$, and hence, since the cone of roots is convex (cf. Theorem 1.2), we know that there exist $K, E \in \mathcal{K}^{3}$ such that $\mathrm{i},-\mathrm{i},-c, c \geq 0$, are the three roots of $f_{K ; E}^{\omega}(z)$. Then, it is an easy computation to check that Aleksandrov-Fenchel inequalities (3.2) yield, in terms of $c$, the relations $4 \geq 3 c^{2} \geq 16 / 3$, a contradiction. Therefore, $\boldsymbol{\omega}$-polynomials are weakly stable for $n=3$ and, from the monotonicity of the cone of the roots (see Theorem 1.4), also for $n=2$.

Finally, we consider the $\boldsymbol{\omega}$-polynomial $f_{B_{4} ; B_{4}}^{\omega}(z)=\kappa_{4} \sum_{i=0}^{4}\left(\begin{array}{l}4 \\ i\end{array}\right) i^{i} z^{i}$. It can be checked with a computer or by applying the Routh-Hurwitz criterion (see e.g. [14], page 181$)$ that $f_{B_{4} ; B_{4}}^{\omega}(z)$ has a root with positive real part $(\nu \approx 0.03838+0.20807 \mathrm{i})$. The non-stability property for all $n \geq 4$ is deduced again from the monotonicity of the cones (Theorem 1.4).

Thus, using Theorem 1.6, the following result is a direct consequence of the above proposition.

Corollary 5.2. If $n \leq 3$ then, for any log-concave measure $\mu$ on $\mathbb{R}_{\geq 0}$, $\mu$-polynomials are weakly stable.

We remark that this bound for the dimension might be not best possible, since we do not know whether $\boldsymbol{\omega}$-polynomials are $\mu$-polynomials for some log-concave measure $\mu$ on the non-negative real line $\mathbb{R}_{\geq 0}$.

Another particulary interesting $\mu$-polynomial is the (relative) Wills polynomial $f_{K ; E}^{g}(z)$. Its stability can be also characterized.

Proposition 5.2. Relative Wills polynomials $f_{K ; E}^{g}(z)$ are weakly stable if and only if $n \leq 7$.

Proof. It is easy to check, using (3.2), that the stability criterion (4.4) is fulfilled for $n=7$. The weak stability property for all $n \leq 6$ follows from Theorem 1.4. Now we consider $f(z)=\sum_{i=1}^{6}\left(\begin{array}{c}8 \\ i\end{array}\right)\left(1 / \kappa_{i}\right) z^{i}$, which is a relative Wills polynomial $f_{K ; E}^{g}(z)$ 
for some $K, E \in \mathcal{K}^{8}$ (see Lemma 3.2). It can be checked with a computer or by applying the Routh-Hurwitz criterion that $f(z)$ has a root with positive real part, $\nu \approx 0.05244+0.94238 \mathrm{i}$. The non-stability property for $n \geq 8$ is deduced again from Theorem 1.4.

We finish the section noticing an additional property of the relative Wills polynomial. In [9], we studied the properties of the classical Wills polynomial $f_{K ; B_{n}}^{g}(z)$ and, among others, we characterized the cone $\mathcal{R}^{g}\left(n ; B_{n}\right)$ in dimensions $n=2,3$ (see Theorem 1.2 in $[9]): \mathcal{R}^{g}\left(i ; B_{i}\right)=\mathcal{R}^{g}\left(B_{i} ; B_{i}\right), i=2,3$. An analogous argument shows that these cones remain unchanged if any gauge body $E$ is considered.

Proposition 5.3. $\mathcal{R}^{g}(i)=\mathcal{R}^{g}\left(B_{i} ; B_{i}\right), i=2,3$.

\section{References}

[1] Betke, U. And Henk, M.: Intrinsic volumes and lattice points of crosspolytopes. Monatsh. Math. 115 (1993), no. 1-2, 27-33.

[2] BisgandD, T. M. And SAsváRI, Z.: Characteristic functions and moment sequences. Positive definiteness in Probability, Nova Science Publishers, Huntington, NY, 2000.

[3] Casella, G. And Berger, R. L.: Statistical inference. The Wadsworth \& Brooks/Cole Statistics/Probability Series. Wadsworth \& Brooks/Cole Advanced Books \& Software, Pacific Grove, CA, 1990.

[4] Gruber, P. M.: Convex and discrete geometry. Grundlehren der Mathematischen Wissenschaften 336, Springer, Berlin, 2007.

[5] Hadwiger, H.: Das Wills'sche Funktional. Monatsh. Math. 79 (1975), 213-221.

[6] Hadwiger, H.: Gitterpunktanzahl im Simplex und Wills'sche Vermutung. Math. Ann. 239 (1979), no. 3, 271-288.

[7] Henk, M. and Hernández Cifre, M. A.: On the location of roots of Steiner polynomials. Bull. Braz. Math. Soc. (N. S.) 42 (2011), no. 1, 153-170.

[8] Henk, M., Hernández Cifre, M. A. and Saorín, E.: Steiner polynomials via ultra-logconcave sequences. Commun. Contemp. Math. 14 (2012), no. 6, 1-16.

[9] Hernández Cifre, M. A. and Yepes Nicolás, J.: On the roots of the Wills functional. J. Math. Anal. Appl. 401 (2013), no. 2, 733-742.

[10] Kampf, J.: On weighted parallel volumes. Beiträge Algebra Geom. 50 (2009), no. 2, $495-519$.

[11] Katkova, O. M. And Vishnyakova, A. M.: A sufficient condition for a polynomial to be stable. J. Math. Anal. Appl. 347 (2008), no. 1, 81-89.

[12] Kato, T.: Perturbation theory for linear operators. Classics in Mathematics, Springer-Verlag, Berlin, 1995.

[13] Krein, M. G. and Nudelman, A. A.: The Markov moment problem and extremal problems. Ideas and problems of P. L. Chebyshev and A. A. Markov and their further developments. Translations of Mathematical Monographs 50, American Mathematical Society, Providence, RI, 1977.

[14] Marden, M.: Geometry of polynomials. Mathematical Surveys 3, American Mathematical Society, Providence, RI, 1966. 
[15] McMullen, P.: Inequalities between intrinsic volumes. Monatsh. Math. 111 (1991), no. $1,47-53$.

[16] NiE, Y.Y. And XIE, X. K.: New criteria for polynomial stability. IMA J. Math. Control Inform. 4 (1987), no. 1, 1-12.

[17] Schneider, R.: Convex bodies: the Brunn-Minkowski theory. Encyclopedia of Mathematics and its Applcations 44, Cambridge University Press, Cambridge, 1993.

[18] Shephard, G.C.: Inequalities between mixed volumes of convex sets. Mathematika 7 (1960), 125-138.

[19] Vitale, R. A.: The Wills functional and Gaussian processes. Ann. Probab. 24 (1996), no. 4, 2172-2178.

[20] Wills, J. M.: Zur Gitterpunktanzahl konvexer Mengen. Elem. Math. 28 (1973), $57-63$.

[21] WiLLs, J. M.: Nullstellenverteilung zweier konvexgeometrischer Polynome. Beiträge Algebra Geom. 29 (1989), 51-59.

[22] Wills, J. M.: Minkowski's successive minima and the zeros of a convexity-function. Monatsh. Math. 109 (1990), no. 2, 157-164.

Received April 19, 2013.

María A. Hernández Cifre: Departamento de Matemáticas, Universidad de Murcia, Campus de Espinardo, 30100-Murcia, Spain.

E-mail: mhcifre@um.es

Jesús Yepes Nicolás: ICMAT, C/ Nicolás Cabrera 13-15, Campus de Cantoblanco, UAM, 28049 Madrid.

E-mail: jesus.yepes@icmat.es

Supported by MINECO-FEDER project MTM2012-34037 and by "Programa de Ayudas a Grupos de Excelencia de la Región de Murcia", Fundación Séneca, 04540/GERM/06. 